\title{
Bend Loss, Tapering, and Cladding-Mode Coupling in Single-Mode Fibers
}

\author{
John D. Love and Céline Durniak
}

\begin{abstract}
A new approach to the physical description and quantification of total bend loss in arbitrarily bent single-mode fiber is based on the coupling of the fundamental mode to cladding modes caused by curvature change. A criterion is derived that limits the maximum allowable rate of change of curvature to reduce bend loss to almost arbitrarily low levels regardless of the maximum curvature, provided only that the coating index is sufficiently smaller than the cladding index. This criterion is less demanding for pretapered fibers or fibers with a thinner cladding.
\end{abstract}

Index Terms-Coupled mode analysis, optical waveguide losses, optical waveguide theory, waveguide bends.

\section{INTRODUCTION}

W ITH THE development of single-mode fibers in the 1970s for application to optical communication systems, the determination of optical power loss from bent single-mode fiber became an immediate problem of practical interest and has received considerable attention over the last three decades with a plethora of theoretical methods being introduced to quantify this loss, e.g., [1], [2]. Generally speaking, the overall loss from the bent fiber has been described in terms of two distinct physical contributions. First, there is a transition loss component that is associated with the rapid or abrupt change in curvature from zero for the straight fiber to a finite value for the bent fiber. This loss can be quantified using the overlap between the fundamental mode field on the straight fiber and the offset field on the bent fiber. Second, there is a pure bend loss component that is associated with radiation from the fiber assuming a constant bend radius. The corresponding loss formula associated with the latter has been derived by a number of different approaches [3], [4] and has been shown to be consistent with experimental measurements of bend loss [5]-[9].

In this letter, we introduce an alternative description of bend loss by following the evolution of the fundamental mode and its power along the fiber with the change in curvature. The emphasis here is on minimizing total bend loss by ensuring that the fundamental mode propagates along the bend approximately adiabatically with minimal coupling to any cladding mode. This comprehensive description also avoids the need to delineate between transition and pure bend loss. We derive a criterion that

Manuscript received March 27, 2007; revised May 24, 2007. This work was supported by the Australian Research Council under ARC Discovery Grant DP0452918.

The authors are with the Optical Sciences Group, Research School of Physical Sciences and Engineering, Australian National University, Canberra ACT 0200, Australia (e-mail: John.D.Love@anu.edu.au).

Digital Object Identifier 10.1109/LPT.2007.902246 provides an approximate upper bound on the rate of change of curvature for a given curvature. Minimizing bend loss also requires the real index of the fiber coating to be sufficiently far below that of the fiber cladding to ensure that there is negligible absorption of the core and cladding modal fields by the coating.

\section{ANALYTICAL APPROACH}

Since bend loss occurs predominantly in the plane of a bent fiber, we work with the well-established model of bend loss that first models the section of fiber in this plane by a bent slab waveguide, and then uses a conformal transformation [10] to transform the bent waveguide into a straight waveguide with a tilted profile, the angle of tilt increasing with decreasing bend radius [11], [12]. Using this model, it is possible to calculate the solution of the scalar wave equation analytically in each layer and represent the scalar electric field in terms of a superposition of Airy functions. Because of the relatively large index difference between the cladding and coating, we do not take account of either absorption or radiation loss over the range of bend radius considered here. Hence, coupling between the fundamental mode and cladding modes is the only possible loss mechanism and this can occur only when there is a change in the curvature or profile tilt along the waveguide.

A set of coupled mode equations and a simple criterion were derived to quantify the loss between the fundamental and cladding modes. As in [13], in the weak power regime, the loss from the fundamental mode per half-beat length depends on the critical rate of change of the bend radius with the distance along the waveguide defined by

$$
\begin{aligned}
\left.\frac{d R}{d z}\right|_{c}= & \frac{-\left(n_{1}-n_{2}\right)^{2} R^{2}}{\lambda n_{\mathrm{co}}} \\
& \times \frac{\left[\int_{-\rho_{\mathrm{cl}}}^{\rho_{\mathrm{cl}}} \psi_{1}^{2} /(R+x) d x \int_{-\rho_{\mathrm{cl}}}^{\rho_{\mathrm{cl}}} \psi_{2}^{2} /(R+x) d x\right]^{1 / 2}}{\int_{-\rho_{\mathrm{cl}}}^{\rho_{\mathrm{cl}}} \psi_{1} \psi_{2} x /(R+x) d x}
\end{aligned}
$$

where $x$ is the transverse coordinate, $R$ is the bend radius, the subscripts 1 and 2 are, respectively, associated to the $\mathrm{TE}_{1}$ and $\mathrm{TE}_{2}$ modes, $n_{i}, i=1,2$ is the effective index of the mode $\mathrm{TE}_{i}, \psi_{i}$ is the solution of the wave equation for the $\mathrm{TE}_{i}$ mode, and $\rho_{\mathrm{cl}}$ is the cladding half-width.

The coupling is considered negligible if the rate of change of the bend radius is smaller than the critical value defined by (1). Therefore, under this condition, the propagation along the bent waveguide will be approximately adiabatic. 

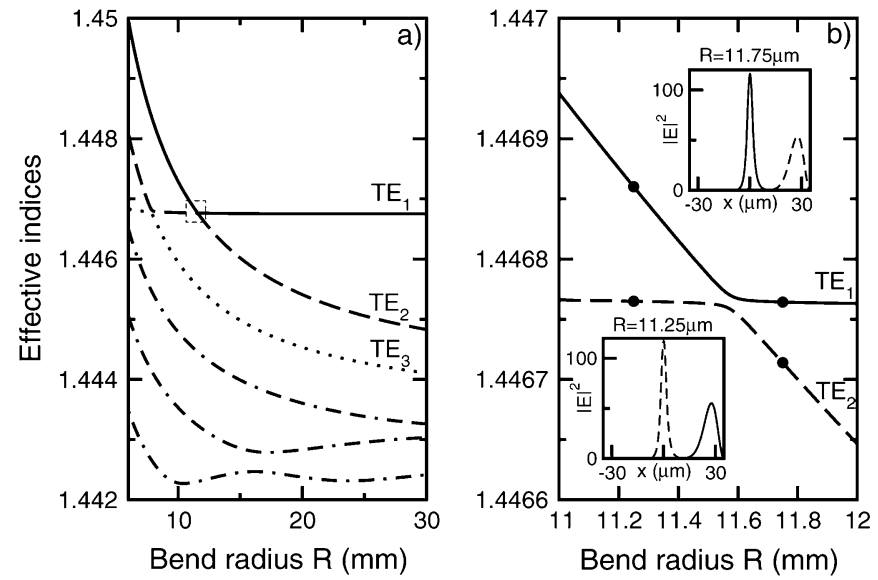

Fig. 1. (a) Effective index values for the first six modes of the bent waveguide as a function of bend radius. (b) Zoom of the evolution of the effective indexes and transverse mode profiles of the first two modes.

\section{RESULTS}

We study the TE modes of a bent slab waveguide consisting of a homogeneous core and a homogeneous finite cladding and for simplicity without a coating so the cladding is surrounded by air. At a source wavelength $\lambda$ is $1.55 \mu \mathrm{m}$, the core has uniform index $n_{\mathrm{co}}=1.4507$ and half-width $\rho_{\mathrm{co}}=1.6 \mu \mathrm{m}$, the cladding half-width is equal to $\rho_{\mathrm{cl}}=35 \mu \mathrm{m}$ and uniform index $n_{\mathrm{cl}}=1.444$. We determine the effective indexes of the core and cladding modes in the weak guidance approximation from the eigenvalue equation, which is obtained by matching the field and its first derivative at the core-cladding interface and by assuming that the field is zero at the cladding-air interface because of the large index difference.

The effective index values for the first six TE modes of the bent waveguide are plotted in Fig. 1 against $R$, the local radius of curvature, varying between 6 and $30 \mathrm{~mm}$. This figure shows that as the curvature increases ( $R$ decreases), successive higher order, i.e., lower effective index modes of the waveguide essentially swap fields when their effective indexes are almost identical. For example, the $\mathrm{TE}_{1}$ mode in Fig. 1 is the lowest order mode and its field has the characteristic Gaussian-shaped-field of the fundamental mode for $R$ larger than $11.55 \mathrm{~mm}$. As $R$ decreases, its effective index becomes close to that of the $\mathrm{TE}_{2}$ mode and the two are nearly equal at $R$ around $11.55 \mathrm{~mm}$. For $R<11.55 \mathrm{~mm}$, the single-peak field becomes the field of the $\mathrm{TE}_{2}$ mode and for a bend radius of $8 \mathrm{~mm}$ where the effective indexes of $\mathrm{TE}_{2}$ and $\mathrm{TE}_{3}$ modes are almost equal, the single-peak field moves again to the $\mathrm{TE}_{3}$ mode, and so on.

As curvature change breaks the rotational invariance of the guide, optical power couples between the core fundamental mode and various cladding modes of the same or different symmetry. The coupling will occur predominantly between modes with the closest propagation constants and is, therefore, strongest in the region where the propagation constants are closest as in Fig. 1, i.e., between the $\mathrm{TE}_{1}$ and $\mathrm{TE}_{2}$ modes. The critical variation of the bend radius with the propagation distance along the curved waveguide (1) is plotted as a function of the bend radius $R$ in Fig. 2. Under the curve, the propagation
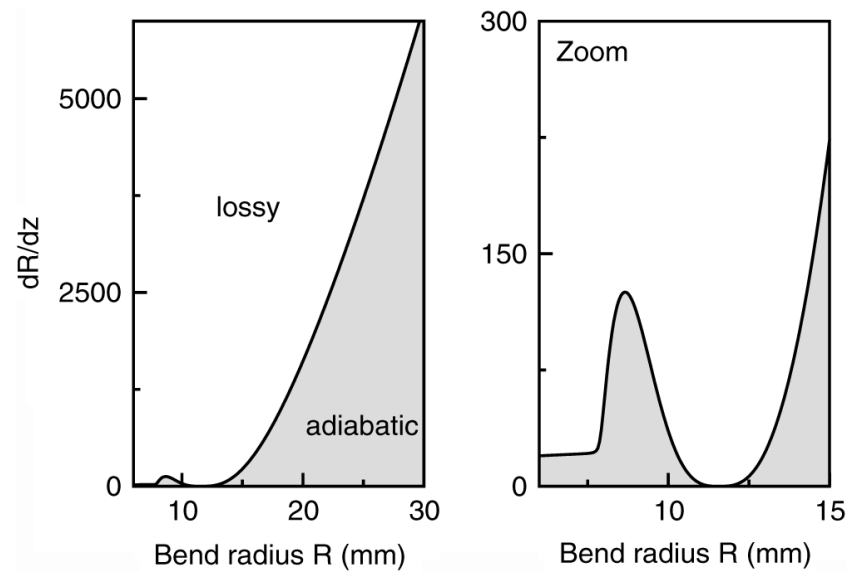

Fig. 2. Delineation curve for the rate of change of bend radius along the waveguide as a function of the bend radius. The gray area corresponds to the adiabatic region.
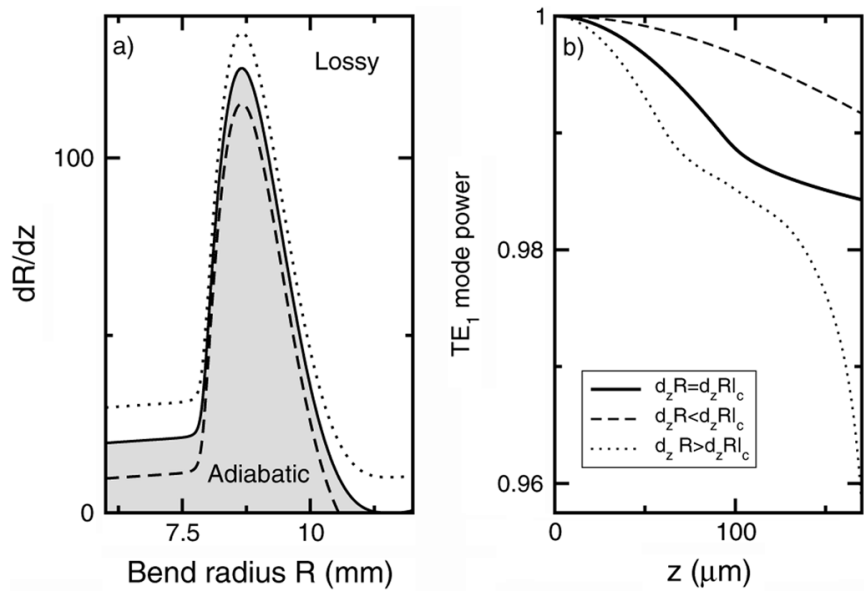

Fig. 3. (a) Rate of change of the bend radius along the bent waveguide axis $z$ as a function of the bend radius for three different configurations. (b) Fundamental mode power evolution along the propagation distance for three different configurations. The solid line is for the critical case, the dotted and dashed lines for $d R / d z$, respectively, above and below the critical case.

is approximately adiabatic. The delineation curve is characterized by a minimum that corresponds to the closest separation between the $\mathrm{TE}_{1}$ and $\mathrm{TE}_{2}$ effective index curves [see Fig. 1(b)]. This is analogous to the approximately adiabatic tapering of single-mode $W$ - and depressed-cladding fibers, where it has been shown [14] that there is a critical tapered core radius at which the maximum permissible local taper angle has a cusp-like minimum. These results also show that more rapid changes of the rate of change of bend radius can be tolerated at larger bend radii.

We solved the corresponding coupled-mode equations [1] in the three cases, with a rate of change of the bend radius respectively equal to, above, and below the critical value $d R /\left.d z\right|_{c}$ given by (1) [see Fig. 3(a)]. An additive difference for the three curves was used to demonstrate the sensitivity of the plots to small variations in the rate of change of bend radius. The evolution of the $\mathrm{TE}_{1}$ mode power along propagation distance $z$ is plotted in Fig. 3. If the rate of change of the bend radius lies below the delineation curve, the waveguide is less lossy than for the two other configurations. For a propagation distance of 

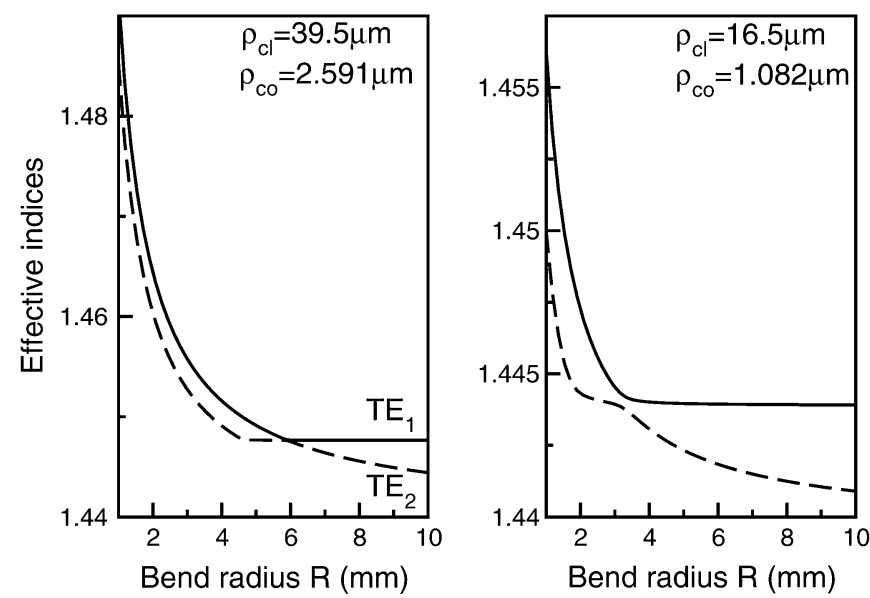

Fig. 4. Plots of effective index of the $\mathrm{TE}_{1}$ and $\mathrm{TE}_{2}$ modes as a function of bend radius for decreasing cladding and core thicknesses.

$170 \mu \mathrm{m}$, the loss of $\mathrm{TE}_{1}$ mode is $0.04,0.07$, and $0.19 \mathrm{~dB}$ for a rate of change of bend radius respectively smaller than, equal to, and larger than the critical value.

If we bend a waveguide such that the rate of change of the bend radius follows the critical rate defined by (1) along the waveguide, the loss of fundamental mode power in the bent waveguide is more sensitive to change in curvature when the coupling between modes is maximum, i.e., for bend radius in the range 11.4-11.7 $\mathrm{mm}$. In this range, the shape of the bent waveguide will be similar to a spiral. Furthermore, the critical rate of change of bend radius increases rapidly with increasing cladding width.

\section{TAPERING AND BENDING}

It is well known that if the coating is removed from a singlemode fiber and it is then pretapered sufficiently by heating and drawing approximately adiabatically from a nominal $125-\mu \mathrm{m}$ overall diameter down to around 10 - to $20-\mu \mathrm{m}$ overall diameter, the waist region can be bent into a very tight bend radius compared to the untapered fiber and without incurring significant bend loss [15]. In this situation, the core fundamental mode field spreads farther into the cladding with reducing core and fiber diameter until in the waist region it is guided predominantly by the cladding-air interface. The $V$-value in the waist region is proportional to both the numerical aperture between glass and air and the cladding radius and is, therefore, relatively large compared to the core-cladding value for the nontapered fiber. If the standard pure bend loss formula is modified accordingly, it correctly predicts negligible bend loss by radiation into air down to bend radii smaller than a millimeter, but it does not account for the absence of transition loss.

Our understanding of mode coupling due to curvature provides insight into the transition loss. As the fiber diameter decreases with tapering, the fundamental and second mode effective indexes move further apart at larger bend radius, as shown in
Fig. 4. Further, the same plots show that the bend radius where the effective indexes of the first and second modes are closest reduces with decreasing bend radius and that the effective index separation at this position increases rapidly. Hence, mode coupling is significantly reduced.

\section{CONCLUSION}

We have shown that, by regarding bend loss from the fundamental mode of a single-mode fiber with a lower-index coating as a cladding-mode coupling process, a simple criterion can be devised that determines the maximum acceptable rate of change of curvature at every position along the fiber to ensure that bend loss is minimized. This insight also provides a strategy for reducing the minimum bend radius in, e.g., fiber organizers. Our description also provides insight into the almost total absence of bend loss when a bare fiber is tapered prior to bending to extremely small bend radius.

\section{REFERENCES}

[1] A. W. Snyder and J. D. Love, Optical Waveguide Theory. London, U.K.: Chapman \& Hall, 1983.

[2] R. C. Gauthier and C. Ross, "Theoretical and experimental considerations for a single-mode fiber-optic bend-type sensor," Appl. Opt., vol. 36, no. 25, pp. 6264-6273, Sep. 1997.

[3] D. Marcuse, "Bend loss of slab and fiber modes computed with diffraction theory," IEEE J. Quantum Electron., vol. 29, no. 12, pp. 2957-2961, Dec. 1993.

[4] H. Vendeltorp-Pommer and J. H. Povlsen, "Bending loss and field distributions in a bent fibre calculated with a beam propagation method," Opt. Commun., vol. 75, no. 1, pp. 25-28, Feb. 1990.

[5] Y. Murakami and H. Tsuchiya, "Bending losses of coated single-mode optical fibers," IEEE J. Quantum Electron., vol. 14, no. 7, pp. 495-501, Jul. 1978.

[6] I. Valiente and C. Vassalo, "New formalism for bending losses in coated single-mode optical fibres," Electron. Lett., vol. 25, no. 22, pp. 1544-1545, Oct. 1989.

[7] H. Renner, "Bending losses of coated single-mode fibers: A simple approach," J. Lightw. Technol., vol. 10, no. 5, pp. 544-551, May 1992.

[8] L. Faustini and G. Martini, "Bend loss in single-mode fibers," J. Lightw. Technol., vol. 15, no. 4, pp. 671-679, Apr. 1997.

[9] Q. Wang, G. Farrel, and T. Freir, "Theoretical and experimental investigations of macro-bend losses for standard single mode fibers," Opt. Express, vol. 13, no. 12, pp. 4476-4484, Jun. 2005.

[10] M. Heiblum and J. H. Harris, "Analysis of curved optical waveguides by conformal transformation," IEEE J. Quantum Electron., vol. 11, no. 2, pp. 75-83, Feb. 1975

[11] J. Lu, S. He, and V. G. Romanov, "A simple and effective method for calculating the bending loss and phase enhancement of a bent planar waveguide," Fiber Integrated Opt., vol. 24, no. 1, pp. 25-36, Jan. 2005.

[12] I. C. Goyal, R. L. Gallawa, and A. K. Ghatak, "Bent planar waveguides and whispering gallery modes: A new method of analysis," J. Lightw. Technol., vol. 8, no. 5, pp. 768-774, May 1990.

[13] J. D. Love et al., "Tapered single-mode fibres and devices. Part 1: Adiabaticity criteria," Inst. Elect. Eng. Proc. J., vol. 138, no. 5, pp. 343-354, Oct. 1991.

[14] W. J. Stewart and J. D. Love, "Design limitation on tapers and couplers in single mode fibres," in 5th Int. Conf. Integrated Opt. and Opt. Fiber Commun., 11th Eur. Conf. Opt. Commun. (IOOC/ECOC'85), Venice, Italy, 1985, pp. 559-562.

[15] T. A. Birks, K. P. Oakley, and C. D. Hussey, "Adiabaticity of miniature loops in tapered single-mode fibre," Elect. Lett., vol. 28, no. 22, pp. 2034-2035, Oct. 1992. 